\title{
Representation Bias, Return Forecast, and Portfolio Selection in the Stock Market of China
}

\author{
Daping Zhao and Yong Fang \\ Academy of Mathematics and Systems Science, Chinese Academy of Sciences, Beijing 100190, China \\ Correspondence should be addressed to Yong Fang; yfang@amss.ac.cn
}

Received 16 December 2013; Revised 9 March 2014; Accepted 11 March 2014; Published 10 April 2014

Academic Editor: Chuangxia Huang

Copyright (c) 2014 D. Zhao and Y. Fang. This is an open access article distributed under the Creative Commons Attribution License, which permits unrestricted use, distribution, and reproduction in any medium, provided the original work is properly cited.

Representation bias means a kind of cognitive tendency, and, for investors, it can affect their behavior in the stock market. Whether the representation bias can help the return forecast and portfolio selection is an interesting problem that is less studied. In this paper, based on the representation bias theory and current markets situation in China, a new hierarchy of stock measurement system is constructed and a corresponding set of criteria is also proposed. On each criterion, we try to measure the influence among stocks with adapted fuzzy AHP. Then the Hausdorff distance is applied to weight and compute the horizontal representation returns. For the forecast returns, according to representation behaviors, there is also a new computation method. Empirical results show that the representation bias information is useful to the return forecast as well as the portfolio selection.

\section{Introduction}

The concept of representation bias is proposed by Tversky and Kahneman $[1,2]$ as a normal behavioral characteristic in financial decisions. They believe that the "representation heuristic" badly affects people's decisions during their opinions building and reasoning. DeBondt and Thaler [3] argue that there exists an overreaction that, after correcting the probabilities, investors may overweight the newly obtained information. As for the investors' behaviors in the security market, Fuller [4] defines one of them as representation bias that may mislead the investors to believe they have already processed the information correctly right before they make a decision. In general, there are two kinds of representation biases: horizontal representation bias and vertical representation bias [5]. According to Zhang [6], the horizontal bias means people have a tendency to classify one thing with its analogues and forecast the thing in the future according to its similarities. Meanwhile, the vertical bias implies that, in financial markets, people easily tend to judge or forecast a stock according to its own history records.

As for the effects behavioral bias might bring to the financial market, many scholars have done some interesting research. In the Chicago Stock Exchange market, Shefrin and Statman's tests show that investors' behavioral bias can significantly and short-termly affect the afternoon stock prices [7]. According to Coval and Shumway [8], prices set by loss-averse traders are reversed significantly and more quickly than those set by unbiased traders. In recent years, behavioral investment portfolio theory has been applied to derive the behavioral investment portfolio frontier and also used for the portfolio selection problem. In this field, how the behavioral bias affects the decision making is focused on by researchers. Chira et al. [9] take experiments with the students in college, and then they analyze the effects of different behavioral biases on financial decisions. Xu et al. [10] extend Tversky's model based on investors' representation bias and under the frame of maximizing the utility; then they examine it with the vertical representation bias as an example. Zhao and Fang [11] propose a computation method of both the vertical and horizontal representation bias returns and try to find if the representation information can help return forecast in financial market.

In the asset allocation, as for the measurement of the subjective things, such as behaviors and emotions, Saaty et al. [12] originally use the AHP to deal with financial problems. Then, with the development of financial theory, complex financial system draws much attention. And the fuzzy theory and methods, which, compared with the traditional ones, are less subjective and can characterize the fuzzy investment 
environment and process better, are gradually put into computation. Enea and Piazza [13] combine fuzzy theory and AHP method together and put forward fuzzy AHP, but they do not solve some problems with special values. Based on their work, Tiryaki and Ahlatcioglu [14] apply an adapted method which solves the zero-value problem to Turkey's stock market, and the investment decisions are made with the mean-variance model. However, the optimal investment weights are not shown. According to [11], the adapted fuzzy analytic hierarchy process method is firstly used to measure horizontal representation bias. The work is based on the consideration that, in the complex financial market, the vivid path of how the representation bias affects the investor's decisions is unknown yet. Although before investors make investment decisions they would evaluate the market, they probably will not calculate the things with specific AHP or some methods so strictly and accurately. In other words, it is like a fuzzy process.

This paper can be seen as an updated version of our last paper mentioned above. This paper follows the main thoughts of how to measure the effect of the horizontal and vertical representation bias on the stock returns, but, instead, considering the current financial environment in China and the related policies, we renovate the evaluation system with hierarchy, criterion, and weights. In the computation part, we apply Hausdorff distance to deal with the weighting problems. According to the situation that the investors' vertical representation bias may influence the expectation of the returns in the future, we propose another method of weighting with the degree of matching the stock's historical data and its present trend and overcome the problem of zerodenominator. Then we take empirical experiments with the data in Chinese stock market, and the results are acceptable. And the new method is also tested empirically, and we compare it with Chira et al.'s method in [9]. At last, we put the forecast returns into a behavior investment portfolio selection model and show the effective frontiers, which suggest that representation bias can help the returns forecast and optimize the portfolio selection to a certain extent.

This paper is organized as follows. In the next section we state a fuzzy measurement about the representation behaviors and a utility model. In Section 3, we apply the methods with an empirical experiment and discuss the computational results. We end up the paper with a summary of conclusions in Section 4.

\section{Methods}

2.1. Representation Bias and Returns. In general, there are two kinds of representation biases: horizontal bias and vertical bias. The horizontal representation bias implies a kind of behavior that people tend to classify one thing with other similar things and forecast it following its similar things' rules. The vertical representation bias signifies another behavior or other habits that people tend to easily judge or forecast a thing according to its own history records (see [5]). Xu et al. put forward a method to calculate the vertical and horizontal representation returns; then Zhao and Fang propose a new one (see $[10,11])$. Here we follow their explanations to the representation returns, but we extend the computation in detail.

2.1.1. Horizontal Representation Returns. The horizontal representation return means the returns which investors forecast and compute with horizontal representation bias and information. Taking stocks, for example, the horizontal representation return of a stock is mainly influenced by the other stocks which have similar characteristics, such as stocks of similar industries and of the same fund company. Investors with horizontal representation bias behaviors tend to judge a stock in the light of the situations of the other similar stocks. Therefore, to build a proper stock hierarchy system is vitally important. In this paper, to calculate the horizontal representation bias returns takes two steps as follows.

Step 1 (pick up the initial stocks). Select some stocks to put in the initial portfolio. Take part 3 of this paper, for example; we select 15 stocks and name them as $A_{1}, A_{2}, A_{3}, A_{4}, A_{5}, \ldots, A_{15}$.

Step 2 (weighting and calculating the horizontal representation bias returns). Choose some characteristics of the stocks which the investors care about. Here we divide the indicators into four groups, including the investment environment, company issues, profitability of the stocks, and the investors' objectives. We choose 30 indicators and denote them as $I_{1}, I_{2}, I_{3}, I_{4}, I_{5}, \ldots, I_{30}$.

In recent years, the Chinese government has weakened its regulation to the stock market in some way, and the "invisible hand" has handled more about the market than before. Hence, compared with our former work, here we lightened the weight of the government supervision and raised the weights of industry development and regional economic conditions, according to regional and industry economic performances have been improved significantly. The new stock hierarchy system is as Table 1 shows.

Define

$$
r_{j}^{h}=\sum_{i=1, i \neq j}^{n} \sum_{k=1}^{m} a_{k} r_{i} w_{k, i}
$$

where $r_{j}^{h}$ means the horizontal representation return of stock $j ; r_{i}$ means the returns of the other similar stocks, $i=1, \ldots, n, i \neq j$; and $w_{k, i}$ means the effect factor of stock $i$ compared with the target stock for criterion $k$. On the criterion $k$, if stock $i$ has a great influence on stock $j, w_{k, i}$ will be endowed with a great value. For example, if stock 1 has a bigger impact on stock $j$ than stock 2 (here $j \neq 1,2$ ), then $w_{k, 1}>w_{k, 2} . a_{k}$ means the weight of criterion $k$ in the whole stock hierarchy system, $\sum a_{k}=1$. It is clear that the return of stock $j$ is a kind of weighted sum of the other stock returns.

It can be found that the key to measure horizontal representation return is to calculate the effect factor $w_{k, i}$; then the representation bias is quantified in some way. 
TABLE 1: Hierarchy, criteria, and weights in the stock selection.

\begin{tabular}{|c|c|c|c|}
\hline Hierarchy & Criteria & Weights & Weights notations \\
\hline \multirow{6}{*}{ Investment environment } & Economics $I_{1}$ & 0.2 & $H_{1}$ \\
\hline & Government supervision $I_{2}$ & 0.1 & $\mathrm{H}_{2}$ \\
\hline & Policies $I_{3}$ & 0.15 & $\mathrm{H}_{3}$ \\
\hline & Industry situations $I_{4}$ & 0.325 & $H_{4}$ \\
\hline & Area situations $I_{5}$ & 0.125 & $H_{5}$ \\
\hline & Others $I_{6}$ & 0.1 & $H_{6}$ \\
\hline \multirow{7}{*}{ Company issues } & Issuance time $I_{7}$ & 0.1 & $\mathrm{H}_{7}$ \\
\hline & Issuance area $I_{8}$ & 0.1 & $H_{8}$ \\
\hline & Substantial shareholders $I_{9}$ & 0.15 & $H_{9}$ \\
\hline & Tradable shareholders $I_{10}$ & 0.15 & $H_{10}$ \\
\hline & Company executives $I_{11}$ & 0.2 & $H_{11}$ \\
\hline & Significant matters $I_{12}$ & 0.2 & $H_{12}$ \\
\hline & Others $I_{13}$ & 0.1 & $H_{13}$ \\
\hline \multirow{12}{*}{ Profitability of the stocks } & Stock market segment $I_{14}$ & 0.1 & $H_{14}$ \\
\hline & Market value $I_{15}$ & 0.1 & $H_{15}$ \\
\hline & Coupon value $I_{16}$ & 0.06 & $H_{16}$ \\
\hline & EPS $I_{17}$ & 0.1 & $H_{17}$ \\
\hline & Shareholders' equity $I_{18}$ & 0.06 & $H_{18}$ \\
\hline & Dividends and placing $I_{19}$ & 0.1 & $H_{19}$ \\
\hline & Earnings $I_{20}$ & 0.1 & $H_{20}$ \\
\hline & Volume $I_{21}$ & 0.1 & $H_{21}$ \\
\hline & Risk assessment $I_{22}$ & 0.1 & $H_{22}$ \\
\hline & The growth of the stock $I_{23}$ & 0.1 & $H_{23}$ \\
\hline & ROE $I_{24}$ & 0.08 & $H_{24}$ \\
\hline & Others $I_{25}$ & 0.02 & $H_{25}$ \\
\hline \multirow{5}{*}{ Investors' perspectives } & The chosen fund company $I_{26}$ & 0.2 & $\mathrm{H}_{26}$ \\
\hline & Financial ability $I_{27}$ & 0.2 & $H_{27}$ \\
\hline & Risk tolerance $I_{28}$ & 0.25 & $H_{28}$ \\
\hline & Expectations for returns $I_{29}$ & 0.25 & $H_{29}$ \\
\hline & Others $I_{30}$ & 0.1 & $H_{30}$ \\
\hline
\end{tabular}

Define

$$
w_{k, i}=\frac{\left(1 /\left(1+H_{k, i}-H_{k, j}\right)\right)}{\sum_{i=1}^{n}\left(1 /\left(1+H_{k, i}-H_{k, j}\right)\right)},
$$

where $H_{k, i}$ means the fuzzy value of stock $i$ on criterion $k$, and it is mainly calculated by the adapted fuzzy analytic hierarchy process method. Then it can be put into the measurement of the similarity between stocks. For the distance between fuzzy numbers, we apply the Hausdorff distance (see $[14,15])$. Take the triangle fuzzy numbers $v_{1}=\left(l_{1}, m_{1}, u_{1}\right), v_{2}=\left(l_{2}, m_{2}, u_{2}\right)$, for example. First we define the distance between point $a$ and a fuzzy number $v_{2}$

$$
D\left(a, v_{2}\right)=\min _{b \in v_{2}}\{d(a, b)\},
$$

where $b \in v_{2}$ can be seen as the value of its membership function is greater than 0 , which implies $\mu_{v_{2}}(b)>0$. Then, the distance between two fuzzy numbers is

$$
h\left(v_{1}, v_{2}\right)=\max _{a \in v_{1}}\left\{D\left(a, v_{2}\right)\right\} .
$$

For distance, it should meet the symmetry. Therefore, the Hausdorff distance between two triangle fuzzy numbers is defined as

$$
d_{1,2}=\max \left\{h\left(v_{1}, v_{2}\right), h\left(v_{2}, v_{1}\right)\right\} \text {. }
$$

With the methods above, the effect factor $w_{k, i}$ can be figured out, and then the horizontal representation return is calculated.

2.1.2. Vertical Representation Returns. The vertical representation bias suggests that investors tend to judge or forecast a stock based on its history instead of the other related things. Therefore, we assume that the vertical representation return of a stock is mainly influenced by its own historical data. And the procedures of calculating the vertical representation returns are as shown below.

Step 1 (pick up the initial stocks). Select some stocks to put in the initial portfolios. 
Step 2 (weight and calculate the vertical representation bias returns). Investors with vertical representation behavioral bias focus on a stock's return history and adapt their expectations based on it. For stock, $i=1, \ldots, n$, we choose its historical returns with $N$ periods and denote them as $R_{i, 1}, R_{i, 2}, \ldots, R_{i, N}$. We try to figure out the similarity in the correlation between the historical data and present data of a stock, and, according to that, we weight the historical data of different periods with notations $M_{i, 1}, M_{i, 2}, \ldots, M_{i, N}$. Chira et al. believe the weights of different periods should satisfy $M_{i, 1}<M_{i, 2}<\cdots<M_{i, N}$, which means the longer the period is from now, the less the weight is (see [9]). But we argue that the effect of each period on the present performance is not so appropriate. By their way, we can find that the nearer periods can have more effect on the forecast, which tend to easily weight too much on the late ones, which encourages the fact that the forecast will follow the tendency to a great extent. Therefore, we propose another method to deal with the weighting, and the new way stresses the matching of the history and present. We suppose that when investors find similar history, they are going to learn the history and forecast the future returns based on the learning. Besides, in calculation, we also use the concept of "distance" to handle the weights. And the distance is an absolute value of the minus.

Define the vertical representation return as shown in the following:

$$
r_{i}^{v}=\frac{\sum_{t=1}^{N} R_{i, t} M_{i, t}}{\sum_{t=1}^{N} M_{i, t}},
$$

where $r_{i}^{v}$ is the vertical representation return of stock, $R_{i, t}$ is the historical return of stock $i$ on time $t$, and $M_{i, t}$ is the weight of $R_{i, t}$, which implies the effect of the history on the present. Define

$$
M_{i, t}=\frac{\left(1 /\left(1+\left|D_{i, t}-D_{i, 0}\right|\right)\right)}{\sum_{t=1}^{T}\left(1 /\left(1+\left|D_{i, t}-D_{i, 0}\right|\right)\right)} .
$$

For the present value, we choose the average of the last $T_{0}$ periods as a proxy variable and denote it as $D_{i, 0} . T_{0}$ can be determined by time series regressions of the returns. $D_{i, t}$ is the absolute value of period $t$ minus present for stock $i$, which is like the distance, $t=1, \ldots, T$, since we mainly care about the effects of the past $T$ periods. It should be noted that, in order to avoid the situation that the denominator is 0 , we set up it as the absolute value plus 1 .

2.1.3. Representation Returns. In real life, however, for investors with representation behavioral bias, it is hard to isolate the biases perfectly clearly from each other. Hence here we try to combine the horizontal and vertical representation returns together and build a new measurement as the representation returns. We introduce a horizontal representation bias preference parameter $\alpha$, which is between 0 and 1 .

Define

$$
r_{i}=\alpha r_{i}^{h}+(1-\alpha) r_{i}^{v}, \quad 0 \leq \alpha \leq 1,
$$

where $r_{i}$ is the combined representation return for stock $i$ and $\alpha$ is the horizontal representation bias preference parameter.
From (8), we can see that when $\alpha$ is 1 , it means that the investors totally trust the horizontal representation returns; when $\alpha$ is 0 , it suggests that the investors turn to the vertical representation returns. Here we mainly analyze the forecast errors by. According to (8), we assume that the real return is $\widehat{r}_{i}$, the forecast error of $r_{i}^{h}$ is denoted as $\mu_{1}$, the forecast error of $r_{i}^{v}$ is denoted as $\mu_{2}$, and the forecast error of $r_{i}$ is $\mu$. Then we have

$$
\begin{aligned}
& \mu_{1}=\left|\frac{r_{i}^{h}}{\widehat{r}_{i}}-1\right|, \\
& \mu_{2}=\left|\frac{r_{i}^{v}}{\widehat{r}_{i}}-1\right| \text {, } \\
& \mu=\left|\frac{r_{i}}{\widehat{r}_{i}}-1\right| \\
& =\left|\frac{\alpha r_{i}^{h}+(1-\alpha) r_{i}^{v}}{\widehat{r}_{i}}-1\right| \\
& =\left|\alpha\left(\frac{r_{i}^{h}}{\widehat{r}_{i}}-1\right)+(1-\alpha)\left(\frac{r_{i}^{v}}{\widehat{r}_{i}}-1\right)\right| \\
& {\left[\begin{array}{l}
\alpha \mu_{1}+(1-\alpha) \mu_{2}, \\
\left(\frac{r_{i}^{h}}{\widehat{r}_{i}}-1\right)\left(\frac{r_{i}^{v}}{\widehat{r}_{i}}-1\right) \geq 0 \\
\alpha \mu_{1}-(1-\alpha) \mu_{2},
\end{array}\right.}
\end{aligned}
$$

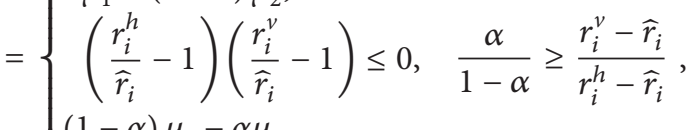

$$
\begin{aligned}
& (1-\alpha) \mu_{2}-\alpha \mu_{1} \text {, } \\
& \left(\frac{r_{i}^{h}}{\widehat{r}_{i}}-1\right)\left(\frac{r_{i}^{v}}{\widehat{r}_{i}}-1\right) \leq 0, \quad \frac{\alpha}{1-\alpha} \leq \frac{r_{i}^{v}-\widehat{r}_{i}}{r_{i}^{h}-\widehat{r}_{i}}
\end{aligned}
$$

By (11), we can see that the forecast error of the representation returns is influenced by the forecast errors of both horizontal and vertical representation returns. And it is also affected by the horizontal representation bias preference parameter $\alpha$. It should be noted that the parameter $\alpha$ depends on the investors' representation preference. If an investor prefers horizontal representation information, then $\alpha$ tends to be greater than 0.5; otherwise, the parameter is smaller.

\subsection{Portfolio Selection Based on Representation Returns and} Prospect Theory. Generally speaking, there are two frameworks about portfolio selection: maximizing the utility and return-risk trade-off. The mean-variance portfolio theory allows investors to minimize the risk with an acceptable return or maximize their expected return with reasonable risk (see $[16,17])$. Nowadays, it has been widely used in real market. However, considering the fact that the traditional mean-variance model might not be proper for the investors' behaviors, we select the portfolio selection model based on prospect theory in the empirical experiment. 
Prospect theory is proposed by Kahneman and Tversky in 1979. In this theory, reference point is a vitally important concept. It is like a benchmark which people tend to use for comparison when they judge something. According to Kahneman and Tversky, they find that investors evaluate an asset mostly depending on the reference point with which the return or loss is compared instead of the real value. In other words, when investors compare with a certain reference level, they care about the relative value even more than the absolute value. When a reference point changes, investors may make totally different decisions. He and Zhou [18] suppose that the reference point is always set as the riskless coupon rate of the bond with long maturity since investors tend to compare the return rate with the coupon rate of bond. In the next section of this paper, we introduce a new parameter showing the reference level.

Assume that there is a single stage model, and the market is free from friction, allowing no short selling. There are $N$ risky assets, and the initial wealth is $W_{0}$. The representation returns are denoted by a vector $r=\left(r_{i}, r_{2}, \ldots, r_{N}\right)$. Define $\theta=\left(\theta_{1}, \theta_{2}, \ldots, \theta_{N}\right)$, in which $\theta_{j}$ is the amount of investment in asset $j$, and $\sum_{j=1}^{N} \theta_{j}=1$. At the end of the investment, the profit is $\widetilde{W}=W_{0}(1+\theta \cdot r)$.

Define the utility of the investors with representation behavioral bias with Fibbo $M$ function. Its classical form is

$$
U(k)=\theta \cdot r-k \sigma^{2}
$$

where $U$ is the utility function and $k$ means the sensitivity of the investors when facing the changes in returns. In addition, we use the prospect theory to measure the changes. There is

$$
\sigma^{2}=v\left(\ln \widetilde{W}-\ln \left(W_{0}\left(1+r_{0}\right)\right)\right) .
$$

Here $v(\cdot)$ is the value function, and $r_{0}$ denotes the investor's reference level. According to Kahneman and Tversky, the loss makes greater impact than returns on the decision making, so the value function is $S$-shaped. In particular, according to Kahneman and Tversky, $v(\cdot)$ can be stated as below:

$$
v(x)= \begin{cases}x^{\gamma}, & x \geq 0 \\ -\lambda(-x)^{\eta}, & x \leq 0\end{cases}
$$

Taking (14) and (13) into (12), there is

$$
U(k, \widetilde{W})= \begin{cases}\theta \cdot r+k\left(\ln \widetilde{W}-\ln \left(W_{0}\left(1+r_{0}\right)\right)\right)^{\gamma}, & r \geq r_{0}, \\ \theta \cdot r-\lambda k\left(\ln \bar{W}-\ln \left(W_{0}\left(1+r_{0}\right)\right)\right)^{\eta}, & r<r_{0} .\end{cases}
$$

According to the rule of maximizing the utility and the market situation in China that there is no short selling, we get the mathematical programming model as follows:

$$
\begin{array}{ll}
\max & U(k, \widetilde{W}) \\
\text { s.t. } & \sum_{j=1}^{N} \theta_{j}=1, \quad \theta_{j} \geq 0 .
\end{array}
$$

\section{Empirical Experiments}

In order to cover different industries and areas, we select 15 stocks from Stock A market of China. The stocks are Poly Real Estate, Daqin Railway, Gree Electric Appliances, ICBC, Gezhouba Dam, Conch Cement, Minsheng Bank, Shandong gold, Sany, Vanke A, Wuliangye, Yunnan Baiyao, Sinopec, Zoomlion, and ZTE. Denote the stock by $A_{1}, A_{2}, A_{3}, A_{4}, A_{5}, \ldots, A_{15}$. All the data is from Wind Database, and the sample is from January 6, 2012, to December 28, 2012, weekly. The returns are calculated with logarithm before computation.

3.1. Calculation of the Horizontal Representation Returns. With the steps of calculating $H_{j i}, W_{i}$ stated in Section 2, the horizontal representation returns are computed as follows.

Step 1. Set the weight $a_{j}$ of every criterion as we show in Table 1.

Step 2. Analyze every indicator, and set the fuzzy pairwise comparison value according to the linguistic importance value: just equal, equally important, weakly important, moderately important, and strongly important. Their triangular fuzzy pairwise comparison values are $(1,1,1),(2 / 3,1,3 / 2)$, $(3 / 2,2,5 / 2),(5 / 2,3,7 / 2)$, and $(7 / 2,4,9 / 2)$.

Step 3. Construct the comparison matrix for every single criterion. Here we show the comparison matrix of the indicator $I_{1}$ tradable shareholders as an example in Table 2.

Step 4. Calculate the $B$ and $C$ matrices, which are 30 in all.

Step 5. Calculate the fuzzy number $H_{k i}$ for every stock on every criterion; then we can get $W_{i}$. Here we show the fuzzy numbers of every stock on criterion $I_{1}$ as an example in Table 3.

As the example shows above, similarly, we can figure out the fuzzy values of the stocks for the other 29 indicators. What is more is that, according to the importance of the different hierarchies, we can also get the different values according to the computation with the similarity between stocks. For example, we assume that the numerical relationship between the four hierarchies is $1: 1: 1: 1$. Then we can standardize the similarities and put them into the calculation of horizontal representation returns. According to Welch and Goyal [19], the average of the historical returns between certain times can be set as benchmarks of forecasting, since the mathematical average without any computation is supposed to contain no information. With this assumption, if the forecast horizontal representation returns perform better, this implies that the horizontal representation acknowledgement provides useful information and can be helpful in the market judgment. In the empirical experiment, we calculate the average with the last four historical numbers as the benchmark and try to rolling forecast the returns in the next four weeks. The results are as Table 4 shows.

From Table 4, we can see that the four forecasts with horizontal representation information all perform better than the 


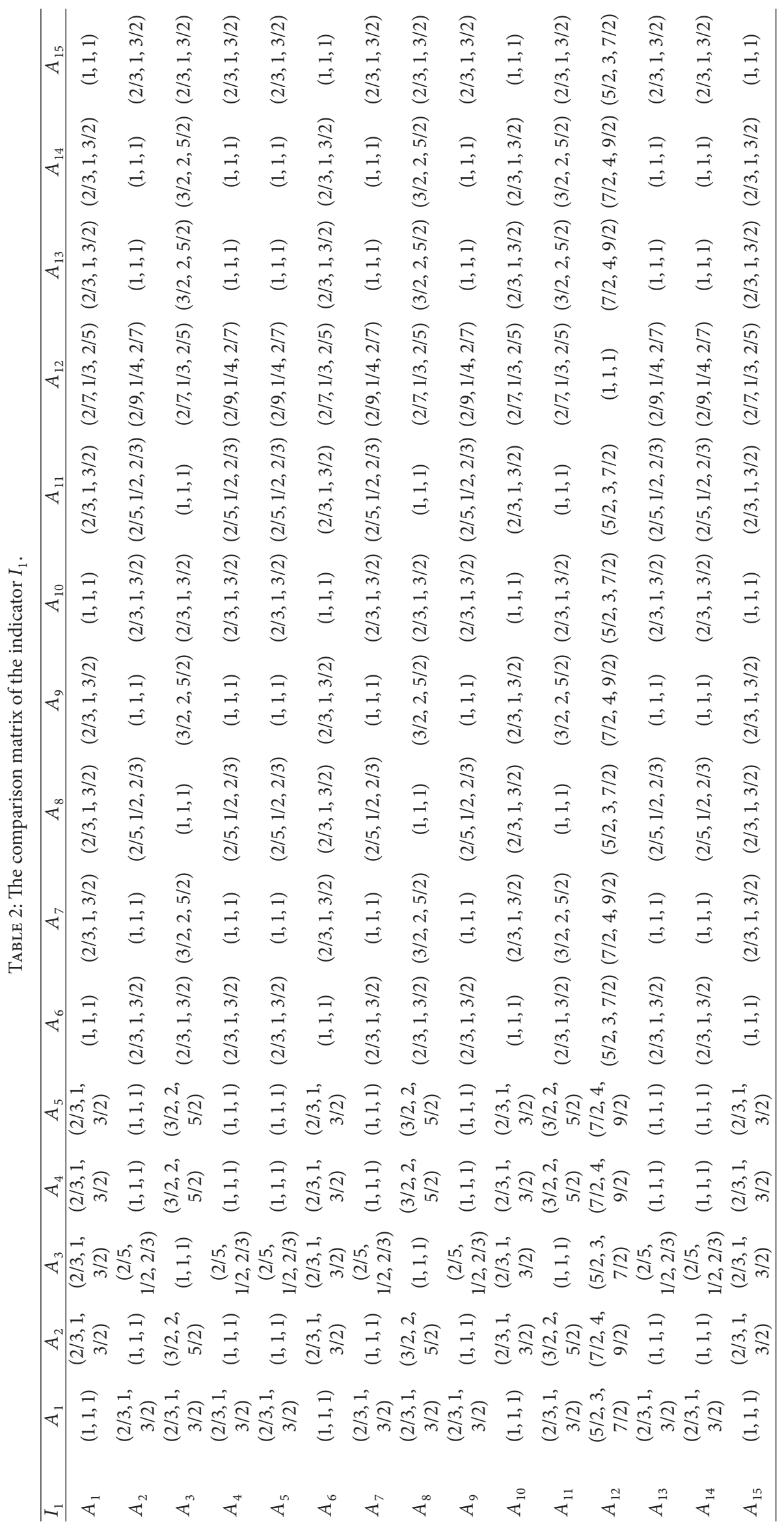


TABLE 3: The fuzzy value of every stock on criteria $I_{1}$.

\begin{tabular}{llcc}
\hline Stocks & \multicolumn{3}{c}{ Fuzzy value $H_{k, i}(i=1, \ldots, n)$} \\
\hline A_1 & 0.0889 & 0.1156 & 0.3048 \\
A_2 & 0.0357 & 0.0478 & 0.1451 \\
A_3 & 0.0424 & 0.0558 & 0.1566 \\
A_4 & 0.0414 & 0.0538 & 0.1566 \\
A_5 & 0.0357 & 0.0478 & 0.1451 \\
A_6 & 0.0536 & 0.0757 & 0.2232 \\
A_7 & 0.0414 & 0.0538 & 0.1566 \\
A_8 & 0.1095 & 0.1156 & 0.3292 \\
A_9 & 0.0427 & 0.0538 & 0.1485 \\
A_10 & 0.0691 & 0.0897 & 0.3292 \\
A_11 & 0.0384 & 0.0505 & 0.1465 \\
A_12 & 0.0406 & 0.0525 & 0.1465 \\
A_13 & 0.0561 & 0.0757 & 0.2324 \\
A_14 & 0.0436 & 0.0558 & 0.1582 \\
A_15 & 0.0436 & 0.0558 & 0.1582 \\
\hline
\end{tabular}

TABLE 4: The error reduction of horizontal representation returns forecast.

\begin{tabular}{lc}
\hline & Error reduction \\
\hline 1 & $9.70 \%$ \\
2 & $20.18 \%$ \\
3 & $87.54 \%$ \\
4 & $1.64 \%$ \\
\hline
\end{tabular}

benchmarks do, and the average error reduction is $29.77 \%$. Considering that the benchmarks are set as containing no information and the new horizontal representation returns look better in forecasting, it is shown in some way that the horizontal representation bias can help forecast the returns. In other words, the investors' representation behaviors can provide useful information in the return forecast. Besides, it should be noted that here we mainly focus on whether the representation behaviors can contain meaningful information instead of the accuracy of the forecast. Since the benchmarks do not forecast very well, the error reductions are great sometimes.

3.2. Calculation of the Vertical Representation Returns. As for the computation and tests of the vertical representation returns, we select the last four months' (sixteen) returns as a sample used in the forecasting. Then we use two methods to weight method in [10] and ours and show the comparison of the results. The assumptions are similar to the horizontal situation stated above, if the forecast returns perform better, which means the information of the vertical representation behaviors can be used in the predictions.

According to the method mentioned in [10], the weights follow an arithmetic sequence, which can make sure that the nearer from now it is, the heavier the weight is, and the weights grow evenly by time. Hence, we set the initial weight as 0.01293 , and the arithmetic is 0.0128 . In this way, the sum
TABLE 5: The errors of vertical representation returns forecast.

\begin{tabular}{ccc}
\hline & $\begin{array}{c}\text { Error reduction with } \\
\text { Xu's method }\end{array}$ & $\begin{array}{c}\text { Error reduction with } \\
\text { our method }\end{array}$ \\
\hline 1 & $-0.75 \%$ & $23.56 \%$ \\
2 & $5.27 \%$ & $5.82 \%$ \\
3 & $16.29 \%$ & $28.52 \%$ \\
4 & $8.83 \%$ & $56.76 \%$ \\
\hline
\end{tabular}

of the last 12 weights is 1 . With (6), the returns of the next 4 periods can be forecasted.

In Section 2, we adapt a method to calculate the vertical representation return, and the method voids the situation of zero-denominator. In the computation part, we first need to find out the lags of the return series by regressions and decide how many periods are needed for them to be picked up as the average will be a proxy variable of the present return. Furthermore, we select the average of the last four periods as the present return level for stock $i$ and denote it as $D_{i, 0}$. Then the weights can be calculated according to (7). At last, we figure out the return forecast results. From Table 5, we can see the results of the two methods. It can be found out that the results with our method are better than those in [10], which suggests that the information of the vertical representation measurements can be useful in the return forecast in some way.

3.3. Results Based on the Portfolio Models of Representation Returns and Prospect Theory. We take the vertical representation returns calculated with our method as an example and put them into the portfolio selection model of prospect theory along with their benchmarks. According to Tversky and Kahneman (see $[1,2]$ ), in the model mentioned in 2.2, the behavioral characteristics are best measured when $\gamma=$ $\eta=0.88, \lambda=2.25$.

We can draw the frontiers of the portfolios with the vertical representation returns $r^{v}$ and the decision variables $\theta$ according to the portfolio selection model. Considering that the portfolio returns are between -0.12 and 0.12 , we divide the interval of $[0,0.12]$ into 20 different levels and calculate every utility value. Then, we get the frontiers with different. The frontiers are as Figure 1 shows.

In Figure 1, the horizontal axis is about the portfolio returns, while the vertical is about the expected utility. All the three frontiers are smooth and downside curves, showing the frontiers when $k=1, k=3$, and $k=5$. We can see clearly that when $k$ is greater, the curve is steeper. That is because when $k$ is greater, the impact of the excess returns on investors' utility is greater and the investors tend to be more sensitive to the changes of returns. By this figure we can also find that, with the return increasing, the utility is decreasing. And this is because when the portfolio returns go up, the expectations of the investors also increase; then the losses from the investment increase, too. We have already known that the losses have $a$ greater impact than the returns on the utility, and then the utility falls down. It should be noted that 


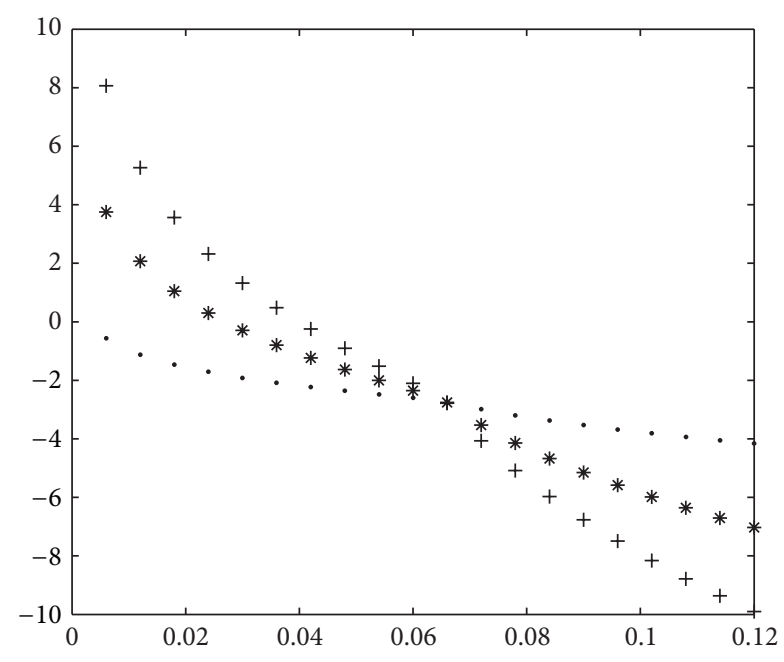

Figure 1: The frontier when $k=1$ (line.), $k=3$ (line $*$ ), and $k=5$ (line + ).

when $k=1$, it means the investors' attitudes to both the profit and the loss are the same.

\section{Conclusions}

In this paper, we continue to focus on what effect the investors' representation behavior may have on the stock returns and investment decisions. First, we update the analytic hierarchy and criterion we constructed before and try to analyze the stock characteristics for investors with horizontal representation behaviors. Then we use the adapted fuzzy AHP to quantify the impact of the criterion on stocks and pay attention to the measurement of the horizontal and vertical representation returns based on the concept of "distance," which implies the similarity between stocks. In this way, the Hausdorff distance is applied to weight and compute the horizontal representation returns. And the problem of zero-denominator in the vertical representation return computation is preliminarily solved.

With the empirical experiments from Chinese stock market, the horizontal representation behavior is proved to be useful to forecast the returns in some way. And the effective frontiers of the behavioral portfolios with vertical representation returns are also shown, which suggest that the representation behaviors can provide useful information to improve the stock returns forecast, and the portfolio frontiers vary according to the investor's attitude to the return changes.

\section{Conflict of Interests}

The authors declare that there is no conflict of interests regarding the publication of this paper.

\section{Acknowledgments}

The authors thank the referees for their thoughtful suggestions and comments. This work was supported by the
National Science Foundation of China (71271201) and the 973 Program (2010CB731400).

\section{References}

[1] A. Tversky and D. Kahneman, "Judgment under uncertainty: heuristics and biases. Biases in judgments reveal some heuristics of thinking under uncertainty," Science, vol. 185, no. 4157, pp. 1124-1131, 1974.

[2] A. Tversky and D. Kahneman, "Advances in prospect theory: cumulative representation of uncertainty," Journal of Risk and Uncertainty, vol. 5, no. 4, pp. 297-323, 1992.

[3] W. F. M. DeBondt and R. Thaler, "Does the stock market overact?" Journal of Finance, vol. 40, no. 3, pp. 793-807, 1985.

[4] R. J. Fuller, "Behavioral finance and the sources of Alpha," Journal of Pension Plan Investing, vol. 2, no. 3, 1998.

[5] R. L. Yu and L. Zhang, Behavioral Finance, Fudan Press, 2nd edition, 2005, Chinese.

[6] C. Zhang, "An important factor on bearish expectations of investors: application of cognitive biases in market forecasts," China Business Research of Economic Theory, vol. 7, pp. 73-74, 2008 (Chinese).

[7] H. Shefrin and M. Statman, "Behavioral portfolio theory," Journal of Financial and Quantitative Analysis, vol. 35, no. 2, pp. 127-151, 2000.

[8] J. D. Coval and T. Shumway, "Do behavioral biases affect prices?” Journal of Finance, vol. 60, no. 1, pp. 1-34, 2005.

[9] I. Chira, M. Adams, and B. Thornton, "Behavioral bias within the decision making process," Journal of Business \& Economics Research, vol. 6, no. 8, pp. 11-20, 2008.

[10] X.-S. Xu, Q. Song, and L.-L. Ma, "Optimal portfolio choice model and empirical analysis based on representation bias," System Engineering Theory and Practice, vol. 32, no. 1, pp. 3440, 2012.

[11] D. P. Zhao and Y. Fang, "Can representation bias help the returns forecast and portfolio selection?" Procedia Computer Science, vol. 17, pp. 603-610, 2013.

[12] T. L. Saaty, P. C. Rogers, and B. Bell, "Portfolio selection through hierarchies," The Journal of Portfolio Management, vol. 6, no. 3, pp. 16-21, 1980.

[13] M. Enea and T. Piazza, "Project selection by constrained fuzzy AHP," Fuzzy Optimization and Decision Making, vol. 3, no. 1, pp. 39-62, 2004.

[14] F. Tiryaki and B. Ahlatcioglu, "Fuzzy portfolio selection using fuzzy analytic hierarchy process," Information Sciences, vol. 179, no. 1-2, pp. 53-69, 2009.

[15] F. Du, W.-K. Shi, and Y. Deng, "New similarity measure of generalized fuzzy numbers," Journal of Shanghai Jiaotong University, vol. 39, no. 4, pp. 614-617, 2005.

[16] H. M. Markowitz, "Portfolio selection," Journal of Finance, vol. 7, no. 2, pp. 77-91, 1952.

[17] X. S. Xu, L. L. Ma, and Y. B. Chen, "Optimal portfolio choice model based on loss aversion," ChInese Journal of Management Science, vol. 15, no. 5, pp. 42-47, 2007 (Chinese).

[18] X. D. He and X. Y. Zhou, "Portfolio choice under cumulative prospect theory: an analytical treatment," Management Science, vol. 57, no. 2, pp. 315-331, 2011.

[19] I. Welch and A. Goyal, "A comprehensive look at the empirical performance of equity premium prediction," Review of Financial Studies, vol. 21, no. 4, pp. 1455-1508, 2008. 


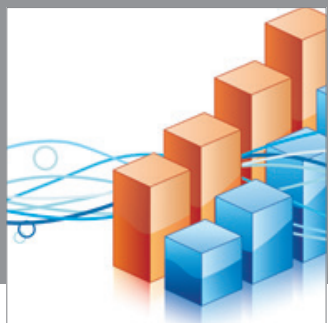

Advances in

Operations Research

mansans

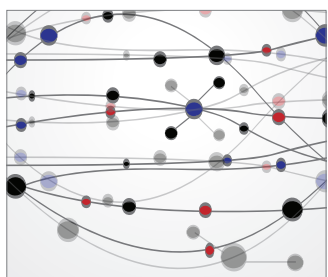

The Scientific World Journal
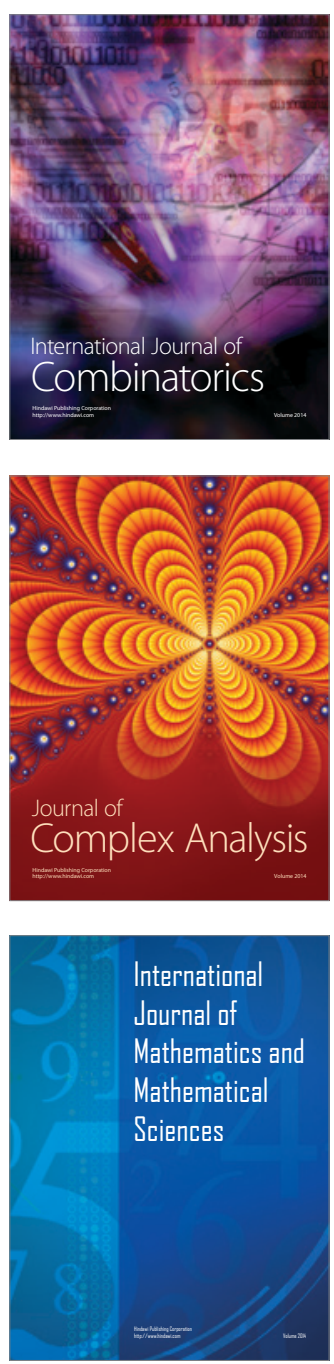
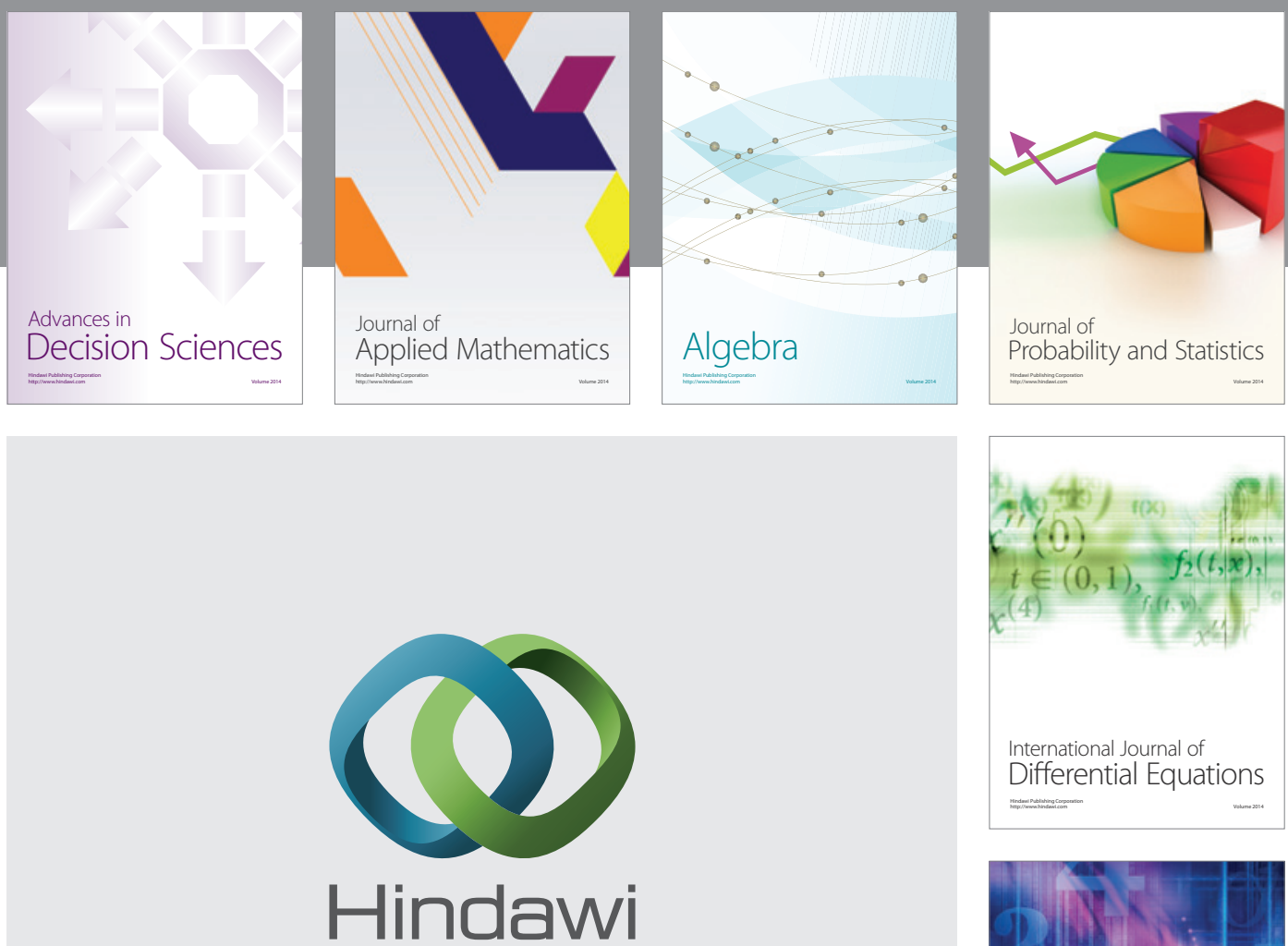

Submit your manuscripts at http://www.hindawi.com
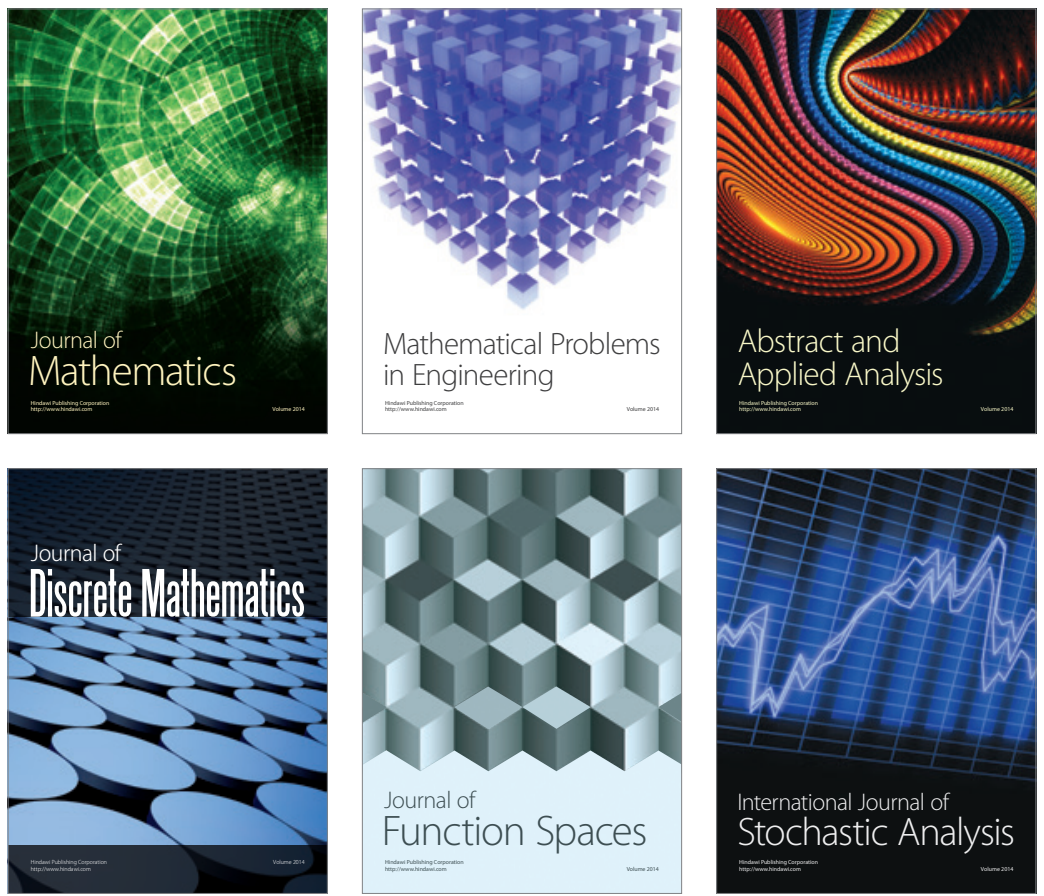

Journal of

Function Spaces

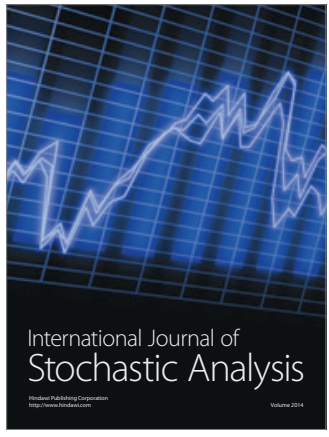

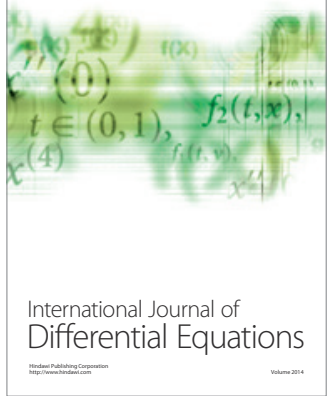
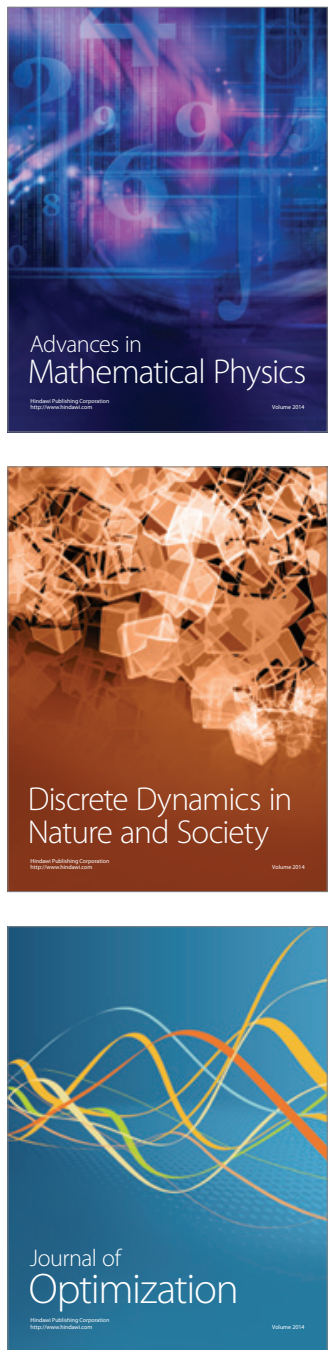